\title{
Effects of Essential Hypertension on coronary Microcirculation: Focus on a Population of Hypertensives Affected by Microvascular Angina
}

\author{
Angelo Quagliana, Salvatore Evola, Oreste Fabio Triolo, Davide Piraino, Giuliana Pace, Giuseppe Riccardo Tona, Vincenzo Sucato, \\ Giuseppe Inga, Rosaria Linda Trovato, Serena Magro, Giuseppe Andolina and Salvatore Novo*
}

Chair and Postgraduate School of Cardiovascular Diseases, Center for Early Diagnosis of Preclinical and multidistrict Atherosclerosis, Department of Internal Medicine and Cardiovascular Diseases, UOC of Cardiology, A. O.U.P. Paolo Giaccone, University of Palermo, Italy

\section{Introduction}

A correlation between essential hypertension and the establishment of myocardial ischemia is nowadays universally accepted [1-3]. Coronary atherosclerosis is deemed to be the most important process through which the capability of coronary district to supply a blood flow consistent with myocardial needs can be impaired, until the onset of an anginal syndrome [4,5]. Hypertension is one of the most widespread cardiovascular risk factors underlying the establishment of an atherosclerotic deterioration of arterial walls; furthermore high blood pressure levels determine a higher risk to incur in a major cardiovascular event, by destabilizing atherosclerotic plaques and boosting platelets tendency to aggregate [6]. Nevertheless, when a cardiac catheterism is performed, in order to unveil the causes of an anginal syndrome according to the positivity of exertional tests and/ or myocardial perfusion scintigraphies, showing up the presence of an ischemic territory, the angiographic visualization of unharmed coronary trees is not infrequent, occurring in an overall percentage of cases settled around 30\% [7]: in this context, a microvascular angina should be hypothesized. In some of these cases, however, the reason for the ischemia is still to be found within epicardial vessels (coronary spasms, myocardial bridges, endothelial dysfunction), whereas in some others none of these suitable causes can be found. In these patients, ischemia can derive from microvascular district, physiological venue of the $80 \%$ of flow resistance. A microvascular dysfunction can be assessed when its regulatory mechanisms are altered, and such abnormality leads to a decrease of coronary flow reserve, without any macroscopic impairment of macroscopic coronary vessels [8]. Nevertheless, its diagnostic definition is usually achieved by a process of exclusion, when an anginal syndrome substained by exertional tests positivity cannot be ascribed to a narrowing of subepicardial vessels' lumen. Such situation has often been associated to the relief of essential hypertension: a considerable percentage of hypertensives, indeed, copes with the experience of chest pain, without any underlying coronary stenosis. The absence of a macrovascular impairment, in these cases, seems to deny the presence of an hypoperfused territory notwithstanding the results of the nuclear imaging tests, having been coronarographies considered, so far, unable to visualize microvascular district. In this study, we verified whether hypertensives' coronaries, seen by performing an angiographic study, are properly definable as normal, even in presence of an overt exertional angina, or if they should rather be barely defined as "macroscopically unharmed", through the clues of a microvascular alteration.

\section{Materials and Methods}

\section{Population}

The analyzed population consists of 173 patients (109 male, 64 female) who underwent heart catheterism in our institute, which showed undamaged coronary trees. Among them, 120 are affected by essential hypertension (average age 62 years, $60 \%$ male), and 47 showed a left Ventricular Hypertrophy, defined by the ultrasound measurement of a septum thickness $>11 \mathrm{~mm}$ during diastole. 53 Normotensives had blood pressure levels under optimal control $(<140 / 90 \mathrm{mmHg}$ for three consecutive supine measurements) without any medical therapy. Diabetic patients were ruled out in order to avoid diabete's influence in the establishment of a systemic microvascular dysfunction. Hypertensives and normotensives showed no substantial differences in terms of prevalence of the main cardiovascular risk factors (Table 1). Among women, none was under estrogen replacement treatment, and only a few of them $(n=5)$ declared they had been under such therapy in the past, making a comparison between them and other women statistically irrelevant. 98 patients presented with chest pain, regressed spontaneously or by the administration of s.l. nitrates. Among them, 32 had a positive Treadmill Test, negative in 9 people and dubious in 2 ; in 66 a myocardial perfusion scintigraphy was performed, which highlighted perfusion defects in $69,7 \%$ of cases, being negative in the others.

\section{Angiographic indices and scintigraphic images}

Several study groups in the recent past have established protocols in order to esteem microvascular functionality in patients who undergo coronary angiography. Gibson et al. introduced Timi Frame Count as a method to study coronary blood flow according to the time spent by the contrast mean to fulfill epicardial vessels, significantly related to the resistance opposed primarily by the microvascular district. Such

\begin{tabular}{|l|l|l|}
\hline CVRF & Hypertensives & Normotensives \\
\hline $\mathrm{n}$ & 120 & 53 \\
\hline Age (yrs) & 62,16 & 59,48 \\
\hline Male Gender (\%) & 60 & 63 \\
\hline Tabagism (\%) & 29 & 35 \\
\hline Familiarity for CVD (\%) & 37 & 32 \\
\hline Hypercholesterolemia (\%) & 34 & 29 \\
\hline Hypertriglyceridemia (\%) & 9 & 11 \\
\hline Obesity (BMl>30 kg/mq) (\%) & 19 & 14 \\
\hline
\end{tabular}

Table 1: Distribution of Cardiovascular risk factors (CVRF) among hypertensives and normotensives.

*Corresponding author: Prof. Salvatore Novo, Chair and Postgraduate School of Cardiovascular Diseases, Center for Early Diagnosis of Preclinical and multidistrict Atherosclerosis, Department of Internal Medicine and Cardiovascular Diseases, UOC of Cardiology, A.O.U.P. Paolo Giaccone, University of Palermo, Viale delle Alpi No 86-90144, Palermo, Italy, E-mail: salvatore.novo@unipa.it

Received December 16, 2011; Accepted January 31, 2012; Published February 05, 2012

Citation: Quagliana A, Evola S, Triolo OF, Piraino D, Pace G, et al. (2012) Effects of Essential Hypertension on coronary Microcirculation: Focus on a Population of Hypertensives Affected by Microvascular Angina. J Clinic Experiment Cardio 3:176. doi:10.4172/2155-9880.1000176

Copyright: (c) 2012 Quagliana A, et al. This is an open-access article distributed under the terms of the Creative Commons Attribution License, which permits unrestricted use, distribution, and reproduction in any medium, provided the original author and source are credited. 
Citation: Quagliana A, Evola S, Triolo OF, Piraino D, Pace G, et al. (2012) Effects of Essential Hypertension on coronary Microcirculation: Focus on a Population of Hypertensives Affected by Microvascular Angina. J Clinic Experiment Cardiol 3:176. doi:10.4172/2155-9880.1000176

index consists of the number of frames recorded for dye to reach a standardized distal landmark of the coronary vessel considered (LAD, CX, RCA), with a correction factor obtained for the LAD by dividing its count by 1.7 , according to its length $[9,10]$. Furthermore, Atmaca Y et al. demonstrated how miscrovascular functionality can be impaired in patients with Syndrome X, through the computation of a new index defined "Myocardial Blush Grade", which evaluates the passage of the contrast mean through microvessels, visualized as a "blush" of the perfused territory, by counting the number of heart cycles required for it to fade out [11]. Moreover, they calculated a Total Myocardial Blush Score, defined as the sum of the MBG of each coronary territory, to define the overall microvascular functionality and verify its possible alterations in patients with dilatative cardiomiopathy and unharmed coronary trees [12]. Atmaca's and Gibson's surveys provided two diagnostic tools, demonstrating their correlation with an impaired microvascular capability to properly control coronary flow resistance. We adopted the same protocols in our hypertnsive patients with normal coronary arteriograms, affected by anginal syndromes, in a bid to determine whether or not a microvascular dysfunction could underlye the onset of such symptoms, by calculating cTFC and MBG and by comparing them to the results of myocardial scintigraphies, in order to identify their eventual congruency. In details, we measured TFC for each major coronary branch and in the mean time assigned a $\mathrm{MBG}=2$ when it took more than three complete cycles for the contrast mean to be washed out of microvascular district, and a score of 3 when it took three cycles or less. As Atmaca et al. assessed, we could get an overview on the whole myocardial microcirculation by adding the MBS we relieved on the three major branches, obtaining the Total Myocardial Blush Score. Moreover, we applied for the first time the same principles to Timi Frame Count, calculating a Total Timi Frame Count as the sum of the three scores, singularly relieved. Angiographic images were separately analyzed by two independent operators, who didn't know the clinical history of patients.

\section{Statistical analysis}

Angiographic data are expressed as means \pm 1 SEM (Standard Error of the Mean, calculated by dividing the Standard Deviation by the square root of the sample size). In order to determine the statistical significance of differences between continuous variables we used a twotailed t-student test. To test statistic correlations between continuous variables we made a linear regression analysis. A p-value $<0,05$ was considered significant. A $\mathrm{k}$ value for inter-observer variability of $0,75-$ for the collection of angiographic data - was achieved.

\section{Results and Discussion}

Although over the last decades several clinical studies have tried to focus on microvascular functionality in patients with chest pain and unharmed coronary trees, the role of its dysfunction in the onset of an ischemic syndrome hasn't been totally understood, yet. The impossibility of visualizing microvessels by angiography is deemed as one of the major causes of such delay, while the common hallmarks of miocardial ischemia, such as stress-induced left ventricular contractile alterations, are often undetectable in these patients. Thus, in clinical practice the diagnosis of microvascular angina is usually assessed by exclusion, rather than by a proper demonstration of microvascular dysfunction [13].

As far as the pathophysiological mechanisms underlying the onset of a microvascular angina are concerned, the possibility for different factors of concurring in the establishment of the dysfunction is nowadays considered the most suitable by a long way. Indeed, while some studies have described, over the last years, structural alterations of small coronary arteries [14], namely smooth muscle hypertrophy, many other study groups didn't get to the same conclusions, and focused on a pattern of differently combined functional abnormalities, such as an altered endothelial functionality [15-17], determining an imbalance in the production of vasomotor mediators in favour of vasoconstrictors like endotheline, and a reduced response of microvessels to endothelium-independent vasodilators (e.g. adenosine) [16,17]. However, among the pathogenetic causes which lead to the onset of a coronary microvascular dysfunction, hypertension has often stood among the others as a likely player of a major role [18]. The results of the comparison of CTFC and MBG, as indices of microvascular functionality, between hypertensives and normotensives are shown in Table 2. It's easy to notice remarkable and significant differences between the two groups, which are ascribable to the effects of hypertension on the establishment of a dysregulation of microvascular resistance and on the reduction of its ability to manage coronary flow reserve according to myocardial needs. Such observation could provide an explanation for the onset of an exertional angina in hypertensives, in spite of the absence of coronary stenosis, being coronary macroscopic indemnity, indeed, unable to clarify the genesis of such symptoms. Thus, it's quite evident how new methods are required to push coronary angiographies' potentialities beyond the mere visualization of epicardial vessels' silhouette, in order to unveil

\begin{tabular}{|l|l|l|l|}
\hline & Hypertensives & Normotensives & Two-tailed t- test \\
\hline cTFC LDA & $27,9 \pm 0,21$ & $20 \pm 0,12$ & $\mathrm{p}<0,001$ \\
\hline cTFC CX & $25,9 \pm 0,10$ & $19,1 \pm 0,12$ & $\mathrm{p}<0,001$ \\
\hline cTFC RCA & $22,9 \pm 0,11$ & $17 \pm 0,08$ & $\mathrm{p}<0,001$ \\
\hline Total TFC & $76,4 \pm 0,10$ & $54,9 \pm 0,14$ & $\mathrm{p}<0,001$ \\
\hline MBG LDA & $2,33 \pm 0,01$ & $2,51 \pm 0,01$ & $\mathrm{P}=0,02$ \\
\hline MBG CX & $2,29 \pm 0,01$ & $2,61 \pm 0,01$ & $\mathrm{p}<0,001$ \\
\hline MBG RCA & $2,19 \pm 0,01$ & $2,61 \pm 0,01$ & $\mathrm{p}<0,001$ \\
\hline Total MBG & $6,8 \pm 0,02$ & $7,75 \pm 0,02$ & $\mathrm{p}<0,001$ \\
\hline
\end{tabular}

Abbreviations: $\mathrm{cTFC}=$ corrected Timi Frame Count, $\mathrm{MBG}=$ Myocardial Blush Grade LDA= Left Descending Anterior, RCA= Right Coronary Artery, $C X=$ Circumflex

Table 2: Timi Frame Count and Myocardial Blush Grade in hypertensives and normotensives.

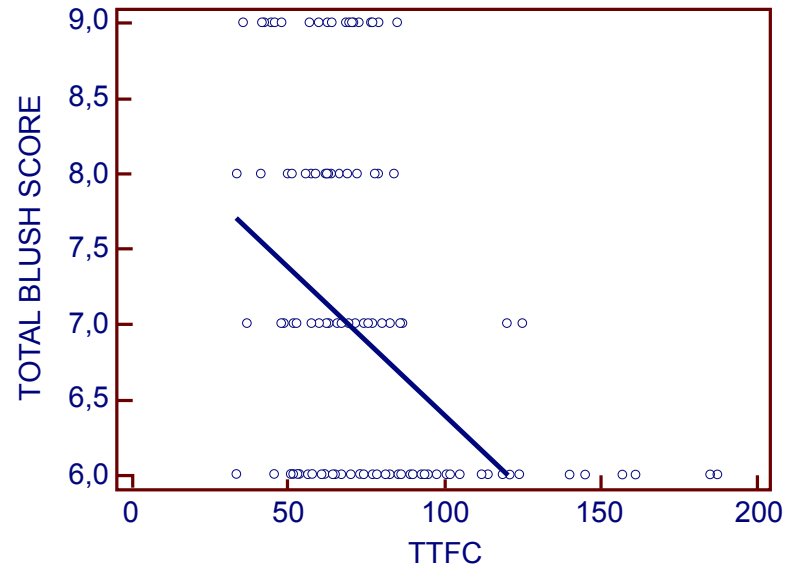

Figure 1: Linear regression analysis: inverse proportionality between TTFC (Total Timi Frame Count) and TMBS (Total Myocardial Blush Score). In hypertensive patients, a worsening of microvascular functionality is demonstrated by the simultaneous alterations of the two parameters, analyzing the modalities through which the contrast mean fulfills coronary vessels during the injection (TTFC) and is washed out from microvessels, afterwards (TMBS). 
Citation: Quagliana A, Evola S, Triolo OF, Piraino D, Pace G, et al. (2012) Effects of Essential Hypertension on coronary Microcirculation: Focus on a Population of Hypertensives Affected by Microvascular Angina. J Clinic Experiment Cardiol 3:176. doi:10.4172/2155-9880.1000176

the presence of a myocardial ischemia even when it is not ascribable to the encroachment of coronary lumen by an atherosclerotic plaque. We compared TTFC to TMBG in a bid to assess their eventual consistency. What we found through a linear regression analysis, shown in Figure 1 , is an inverse proportionality by which the increase of the former, highlighting a slow flow of the contrast mean into the epicardial vessels, goes hand in hand with the reduction of TMBS, which underlines its slow removal from the microvascular territory perfused by the same coronaries. Such correspondence allows us to assess the congruity of the two indices and their agreement in relieving the clues of an altered microvascular functionality, although such data do not permit the identification a proper threshold, whose overcoming would determine the onset of an ischemia and the raise of its related symptoms.

A detailed analysis of patients affected by Left Ventricular Hypertrophy didn't highlight any significant difference between hypertrophyc and non hypertrophic hearts, in terms of microvascular functionality. Such data do not provide an explaination for the higher incidence of anginal syndromes with normal coronary angiograms in patients with LVH $[19,20]$. Nevertheless, in hypertrophic hearts the growth of myocardial tissues is not followed by a contemporary expansion of the vascular network, establishing a disproportion between blood supply and perfused territories' mass [21]. Moreover, there are experimental evidences of an attenuated activity of vasodilating and bradycardia-inducing reflexes in hypertrophic patients, normally activated in response to the rise of intracameral pressure, occurring during an ischemic episode [22], suitable explanations for the higher incidence of ischemic syndromes in hypertensives affected by LVH.

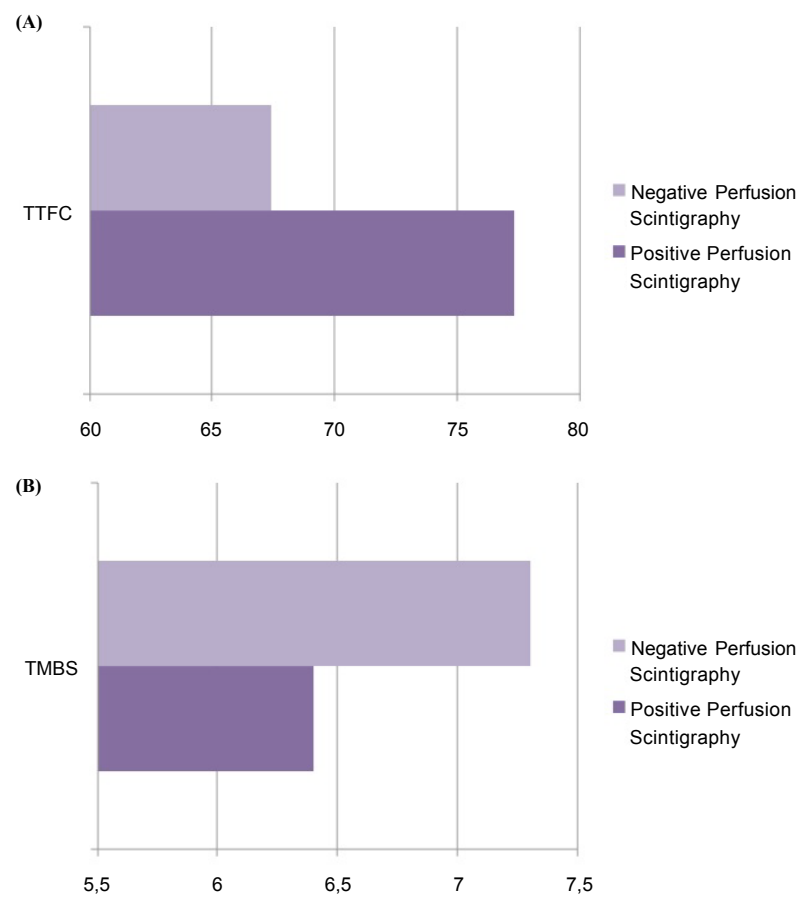

Figure 2A and 2B: Comparison of TTFC ( $P<0,05$; figure $2 A)$ and TMBS ( $p$ $<0,01$; figure $2 B$ ) between patients whose perfusion scintigraphy showed a perfusion defect and patients whose did not; $p$-value $<0,05$. According to the graphs, patients with an ischemia demonstrated through the performance of a myocardial scintigraphy have much worse microvascular functionality indices, in comparison with patients whose nuclear imaging show no ischemic alterations at all.

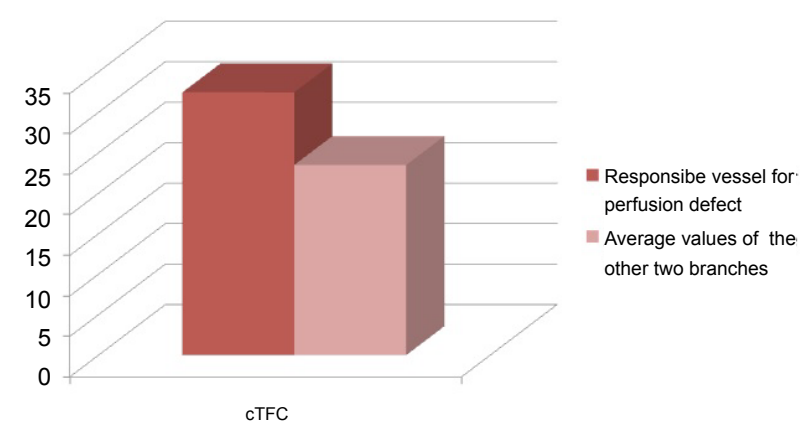

Figure 3: Comparison of cTFC between the vessel responsible for the scintigraphic perfusion defect and the average of the values relieved on the other two major branches; $p$-value $<0.0001$. As shown, heart cath confirms the reliefs of scintigraphic images notwithstanding the indemnity of subepicardial vessels, by showing in the artery responsible for the perfusion defect much worse indices of microvascular functionality in comparison to the average of the other two major branches.

As far as myocardial perfusion scintigraphy is concerned, among hypertensives with a positive nuclear imaging, showing a partially reversible hypoperfused area, far worse angiographic indices were relieved $($ TTFC $=77,27 \pm 0.9$ vs. $67.38 \pm 1.25, \mathrm{p}<0,05$, Figure $2 \mathrm{~A}$; TMBS $=6.4 \pm 0.03$ vs. $7.3 \pm 0.06, p<0,01$, Figure $2 \mathrm{~B})$. Furthermore, in a comparison between the vessel responsible for the perfusion of the scintigraphic defect and an average of the other two vessels, the former's indices were usually significantly worse (namely considering data about cTFC, $32.48+0.68$ vs. $23.5+0.52$, p $<0,0001$, Figure 3 ). Such evidence may be read as a substantial agreement between the two angiographic indices and scintigraphic results, by which patients with a positive nuclear imaging for perfusion defects usually show altered microvascular conditions, despite the indemnity of subepicardial vessels. Much as nuclear sequences are, in such cases, often labelled as "false positives", the opportunity of a microvascular dysfunction, invisible in arteriograms as they are usually meant, should be kept in consideration, in order not to omit the diagnosis of a real ischemia established in spite of the indemnity of macroscopic coronaries [23]. If coronarography is certainly the gold standard technique for the assessment of an atherosclerotic impairment of epicardial vessels, perfusion scintigraphy, by identifying an ischemic area from a metabolic point of view and regardless the causes underlying the onset of the ischemia itself, may fit more the need to define the microvascular genesis of a microvascular anginal syndrome, being coronary silhouette, in such cases, not impaired at all. Nevertheless, being not perfusion scintigraphy routinely included in the diagnostic process of ischemic patients, angiographic sequences can still provide useful informations about coronary blood flow, through the worthwhile computation of the examined indices, able to raise the suspicion of an involvement of microvascular district in the perfusion lacks.

\section{References}

1. Hypertension Control (1997) WHO technical report series 862. World Health Organization, Geneva

2. Williams GH, Malattia vascolare ipertensiva. In: Harrison's Principles of Internal Medicine, 15ma edizione. McGraw-Hill.

3. Agabiti-Rosei E, Muiesan ML (2000) The primary and secondary prevention of hypertensive cardiopathy. Ital Heart J 1: 59-66.

4. Habib Samady H, Parham Eshtehardi P, McDaniel MC, Suo J, Dhawan SS, et al. (2011) Coronary artery wall shear stress is associated with progression and 
Citation: Quagliana A, Evola S, Triolo OF, Piraino D, Pace G, et al. (2012) Effects of Essential Hypertension on coronary Microcirculation: Focus on a Population of Hypertensives Affected by Microvascular Angina. J Clinic Experiment Cardiol 3:176. doi:10.4172/2155-9880.1000176

transformation of atherosclerotic plaque and arterial remodeling in patients with coronary artery disease. Circulation 124: 779-788.

5. Taddei S, Versari D, Virdis A (2002) L'endotelio, fisiopatologia e valore clinico. Monografie di Cardiologia.

6. Fedele F, Romano S (2000) Ipertensione e circolo coronarico. Ital Heart J 1: 13-16.

7. Kephr HG Jr, Vokonas PS, Cohn PF, Gorlin R (1973) The anginal syndrome associated with normal coronary arteriograms. Report of a six years experience. Am J Med 54: 735-742.

8. Yang EH, Lerman A (2005) Angina pectoris with a normal coronary angiogram. Herz 30: 17-25.

9. Gibson CM, Cannon CP, Daley WL, Dodge JT, Alexander B Jr, et al. (1996) TIMI frame count: a quantitative method for assessing coronary artery flow. Circulation 93: 879-888.

10. Gibson CM, Karha J, Giugliano RP, Roe MT, Murphy SA, et al. (2004) Association of the timing of ST-segment resolution with TIMI myocardial perfusion grade in acute myocardial infarction. Am Heart J 147: 847-852.

11. Atmaca Y, Ozdemir AO, Ozdol C, Oguz D, Gulec S, et al. (2005) Angiographic evaluation of myocardial perfusion in patients with syndrome $\mathrm{X}$. Am J Cardiol 96: 803-805.

12. Atmava Y, Duzen V, Ozdol C. Altin T, Tulunay C, et al. (2008) Total blush score: a new index for the assessment of microvascular perfusion in idiopathic dilated cardiomyopathy. Coron Artery Dis 19: 181-185.

13. Lanza GA, Crea F (2010) Primary Coronary microvascular dysfunction. Clinical presentation, pathophysiology and management. Circulation 212: 2317-2325.

14. Opherk D, Zebe H, Weihe E, Mall G, Durr C, et al. (1981) Reduced coronary dilator capacity and ultrastructural changes of the myocardium in patients with angina pectoris but normal coronary arteriograms. Circulation 63: 817-825.
15. Motz W, Vogt M, Rabenau O, Scheler s, Lückhoff A, et al. (1991) Evidence of endothelial dysfunction in coronary resistence vessels in patients with angina pectoris and normal coronary angiograms. Am J Cardiol 68: 996-1003.

16. Chauhan A, Mullins PA, Taylor G, Petch MC, Schofield PM (1997) Both endothelium-dependent and endothelium independent function is impaired in in patients with angina pectoris and normal coronary angiograms. Eur Heart J 18: 60-68.

17. Bottcher M, Botker He, Sonne H, Nielse TT, Czernin J (1999) Endothelium dependent and independent perfusion reserve and the effect of L-arginine on myocardial perfusion in patients with syndrome X. Circulation 99: 1795-1801.

18. Camici PG, Crea F (2007) Coronary microvascular dysfunction. N Eng J Med 356: $830-840$.

19. Novo S, Barbagallo M, Abrignani MG, Nardi E, Di Maria GU, et al. (1997) Increased prevalence of cardiac arrhythmias and transient episodes of myocardial ischemia in hypertensives with left ventricular hypertrophy but without clinical history of coronary heart disease. Am J Hypertens 10: 843-851.

20. Novo S, Abrignani MG, Novo G, Nardi E, Dominguez LJ, et al. (2001) Effects of drug therapy on cardiac arrhythmias and ischemia in hypertensives with LVH Am J Hypertens 14: 637-643.

21. Lembo G, Morisco C, Lanni F, Barbato E, Vecchione C, et al. (1998) Systemic hypertension and coronary artery disease: the link. Am J Cardiol 82: $2 \mathrm{H}-7 \mathrm{H}$.

22. Yoshinaga K, Manabe O, Tamaki N (2011) Physiological assessment of myocardial perfusion using nuclear cardiology would enhance coronary artery disease patient care: which imaging modality is best for evaluation of myocardial ischemia? (SPECT-side). Circ J 75: 713-722.

23. Salerno M, Beller GA (2009) Noninvasive assessment of myocardial perfusion Circ Cardiovasc Imaging 2: 412-424.

\section{Submit your next manuscript and get advantages of OMICS} Group submissions

Unique features:

- User friendly/feasible website-translation of your paper to 50 world's leading languages

Audio Version of published paper

Digital articles to share and explore

Special features:

200 Open Access Journals

15,000 editorial team

21 days rapid review process

Quality and quick editorial, review and publication processing

Indexing at PubMed (partial), Scopus, DOAJ, EBSCO, Index Copernicus and Google Scholar etc

Sharing Option: Social Networking Enabled

- Authors, Reviewers and Editors rewarded with online Scientific Credits

Better discount for your subsequent articles

Submit your manuscript at: www.editorialmanager.com/clinicalgroup 